\title{
Artículos
}

\section{Tecnología, innovación y web social: el valor de la dimensión en la biblioteca pública. El caso de la biblioteca de Muskiz}

\author{
Por Fernando Juárez-Urquijo
}

\begin{abstract}
Resumen: Se hace un repaso de la evolución de las tecnologías informacionales y su relación con la biblioteca desde finales del siglo XX hasta la actualidad y se analiza la respuesta de las bibliotecas frente a los cambios tecnológicos, en especial frente a internet y la web. Estudiamos el caso de una biblioteca pública de pequeña escala, la Biblioteca Municipal de Muskiz. (Bizkaia) y el uso que hace de la web social para ofrecer nuevos servicios bibliotecarios. Este artículo sugiere que el abaratamiento del acceso a la tecnología desplaza la capacidad de innovar desde los centros que poseen recursos para adquirir y gestionar soluciones propietarias hacia las instituciones que modifican sus productos y procesos utilizando servicios web abiertos. Describimos cómo la escala de la biblioteca condiciona el acceso a la tecnología y la sitúa frente a la discontinuidad tecnológica: en el XX favorece a las grandes estructuras y actualmente las penaliza.
\end{abstract}

Palabras clave: Innovación, Web social, Colaboración descentralizada, Cooperación bibliotecaria, Bibliotecas públicas, Biblioteca de Muskiz.

Title: Technology, innovation and the social web: the value of dimension in the public library. The case of the Muskiz library

Abstract: The evolution of information technology and its relationship with libraries is reviewed, from the end of the twentieth century to the present, with a discussion of libaries' response to technological changes, especially the internet and the web. A case study focuses on a small public library in Muskiz (Bizkaia/Biscay province), and its use of the social web to offer new library services. Cheaper access to technology may shift the capacity for innovation, from centres that have resources to acquire and manage proprietary solutions to

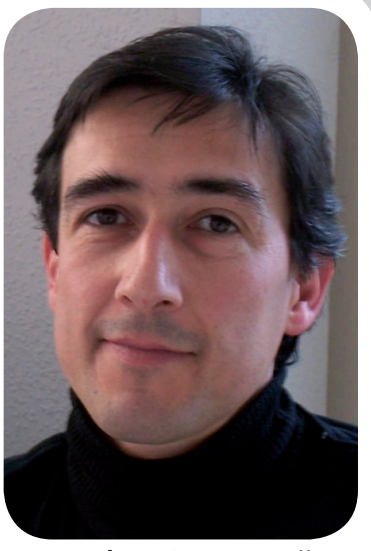

Fernando Juárez-Urquijo es licenciado en geografía e historia (Universidad de Deusto) y master en documentación digital (Universidad Pompeu Fabra). Trabaja en la biblioteca pública de Muskiz desde 1988, desde donde promueve proyectos colaborativos con otras bibliotecas públicas. institutions that modify their products and processes using open web services. We describe how scale affects a library's access to technology and positions it in technological discontinuity: in the 20th century, technology was on the side of larger structures but currently they are penalized.

Keywords: Innovation, Social web, Decentralized collaboration, Library cooperation, Public libraries, Muskiz library.

Juárez-Urquijo, Fernando. “Tecnología, innovación y web social: el valor de la dimensión en la biblioteca pública. El caso de la biblioteca de Muskiz”. En: El profesional de la información, 2008, marzo-abril, v. 17, n. 2, pp. $135-143$.

DOI: 10.3145/epi.2008.mar.02

\section{Introducción}

La tercera edición del Manual de Oslo (OECD, 2005) define la innovación como "la introducción de un nuevo o significativamente mejorado producto (bien o servicio), de un proceso, de un nuevo método de comercialización o de un nuevo método organizativo, en las prácticas internas de la empresa, la organización del lugar de trabajo o las relaciones exteriores".

Aunque la innovación no necesita ser tecnológica, en la actual economía los productos y servicios tienen un elevado componente de tecnología, por lo que habi- tualmente identificamos ambos conceptos. La aparición de una nueva tecnología o el rediseño de otras ya existentes que dan como resultado la desaparición de un producto o servicio que es sustituido por otro, producen la denominada discontinuidad tecnológica. A este fenómeno se le conoce como innovación radical, ya que provoca cambios verdaderamente revolucionarios en la industria por el reemplazo de las tecnologías y por todas las alteraciones que ello implica, en particular en los productos y en los mercados mismos (Sánchez-Novoa, 2002). Dependiendo de las estrategias de respuesta (innovación) de las organizaciones, la discontinuidad 
tecnológica puede ser una amenaza que acabará con su modelo de negocio o una gran oportunidad que le abrirá nuevos mercados.

Innovación, competencia, investigación y desarrollo son conceptos tradicionalmente vinculados al mundo empresarial y asociados al uso de grandes y costosas tecnologías e infraestructuras gestionadas con modelos organizativos fuertemente burocratizados. En este esquema la biblioteca pública se sitúa en el furgón de cola, alejada de los centros de decisión y sin capacidad real para poder implicarse de facto en procesos creativos e innovadores con base tecnológica.

Sin embargo, el desarrollo creciente de tecnologías ligeras, baratas y descentralizadas permite un nuevo modo de aproximación a la innovación en el que la biblioteca pública, habituada a buscar soluciones creativas a su falta estructural de recursos, puede aplicar nuevas formas de gestión y crear productos y servicios innovadores.

\section{"La innovación ahora no depende tanto del poder adquisitivo como de la facilidad de reacción y adaptación ante el cambio tecnológico"}

\section{Innovación, tecnología y biblioteca}

La información es una necesidad básica del ser humano, y al igual que sucede con el resto de bienes y servicios, la tecnología y su evolución inciden en las condiciones de su creación, almacenamiento, gestión y difusión. La aceleración tecnológica de finales del siglo XX y su impacto en los servicios y productos informacionales provocan períodos de discontinuidad tecnológica que motivan una reflexión no exenta de incertidumbre en el mundo bibliotecario, sobre la asimilación y utilización de las tecnologías y sobre el papel de las bibliotecas y sus profesionales (Jantz, 2002). En un entorno muy cambiante la biblioteca debe replantear su "modelo" de servicio y decidir si se enfrenta a una amenaza o si tiene ante sí una oportunidad de futuro.

La capacidad de innovar está directamente relacionada con el acceso a la tecnología, el cual tradicionalmente está muy condicionado por su coste económico. Hay una relación muy directa entre el tamaño de una empresa y su disponibilidad de recursos. En el ámbito bibliotecario la escala de la biblioteca, su tamaño, ha condicionado el acceso a la tecnología y por ende su capacidad de innovar y su posición frente a la discontinuidad tecnológica. Sin embargo la rápida evolución de las tecnologías informacionales y la reducción de sus costes de adquisición ha propiciado que la innovación ahora no dependa tanto del poder adquisitivo como de la facilidad de reacción y adaptación ante el cambio tecnológico.

El paradigma propio de la era industrial estipulaba que la estrategia de respuesta a las nuevas condiciones, la innovación, estaba sujeta al uso de grandes y costosas tecnologías e infraestructuras gestionadas con modelos organizativos fuertemente burocratizados (Freire, 2007). En ese contexto el acceso a la tecnología se situaba en los centros que poseían recursos para adquirir y gestionar soluciones propietarias: grandes bibliotecas con estructura jerárquica vertical y una gran separación entre la toma y la ejecución de decisiones (bibliotecas de gran escala), que disponen de medios para poder implicarse en procesos creativos e innovadores con base tecnológica. Estos centros poseen capacidad para acometer proyectos de envergadura pero necesitan mucho tiempo para tomar y ejecutar decisiones.

En ese modelo la mayoría de las bibliotecas públicas de pequeña escala (aquellas en las que no hay separación entre quien toma y ejecuta las decisiones) y con pocos recursos, no llegaban al umbral tecnológico; son subsidiarias en lo referente a tecnología e innovación y se encuentran en una situación marginal respecto a la toma de iniciativas y decisiones. La innovación en la biblioteca pública llega de arriba a abajo, y la creatividad depende de los recursos disponibles.

El progresivo abaratamiento de los componentes informáticos, la eclosión de internet y el desarrollo de tecnologías ligeras, baratas y descentralizadas reducen progresivamente el umbral de acceso a la tecnología, facilitando la incorporación de nuevos agentes innovadores: bibliotecas de pequeña escala, poco jerarquizadas y muy flexibles para tomar y ejecutar decisiones.

En este nuevo contexto (¿sociedad post-industrial?) la creatividad se "libera" de los costes estructurales y se observa un progresivo desplazamiento de la capacidad de innovar desde los centros que poseen recursos para adquirir y gestionar soluciones propietarias hacia aquellos que modifican sus productos y procesos utilizando servicios web abiertos.

Si en el período industrial el tamaño favorece a las grandes estructuras bibliotecarias, en el contexto actual las penaliza. Se invierte el valor de la escala ante la innovación: cuanto menor sea la distancia entre la toma y la ejecución de las decisiones, mayor flexibilidad habrá para modificar los procesos de producción y generar nuevos y mejores productos; las bibliotecas con centros de decisión/ejecución más alejados, que también se percatan del nuevo escenario, necesitan más tiempo para intentar implementar estrategias de mejora. 
La biblioteca pública se encuentra ante un nuevo escenario en el que es posible, con un bajo coste económico y de aprendizaje, el acceso a una tecnología que permite sustanciar nuevos servicios y aplicar nuevas formas de gestión. En este contexto tecnológico la flexibilidad es una ventaja. Y junto a los tradicionales proyectos arriba-abajo aparecen soluciones "abajo-abajo" impensables anteriormente.

\section{"El tamaño de la biblioteca condiciona su acceso a la tecnología: en el siglo XX se favorecía a las grandes estructuras bibliotecarias pero actualmente las penaliza"}

\section{Evolución tecnológica en una biblioteca pública de pequeña escala: el caso de Muskiz}

No es habitual asociar el concepto de innovación con el de biblioteca pública, no por lo menos en el ámbito en el que se sitúa el objeto de este estudio de Muskiz: una población de 7.100 habitantes con biblioteca pública desde 1987 y una disponibilidad de recursos por debajo de las recomendaciones Ifla ${ }^{2}$ (Mapa de lectura de Euskadi, 2007). La biblioteca es un centro de "pequeña escala": la toma de decisiones y su ejecución es realizada por la misma persona.

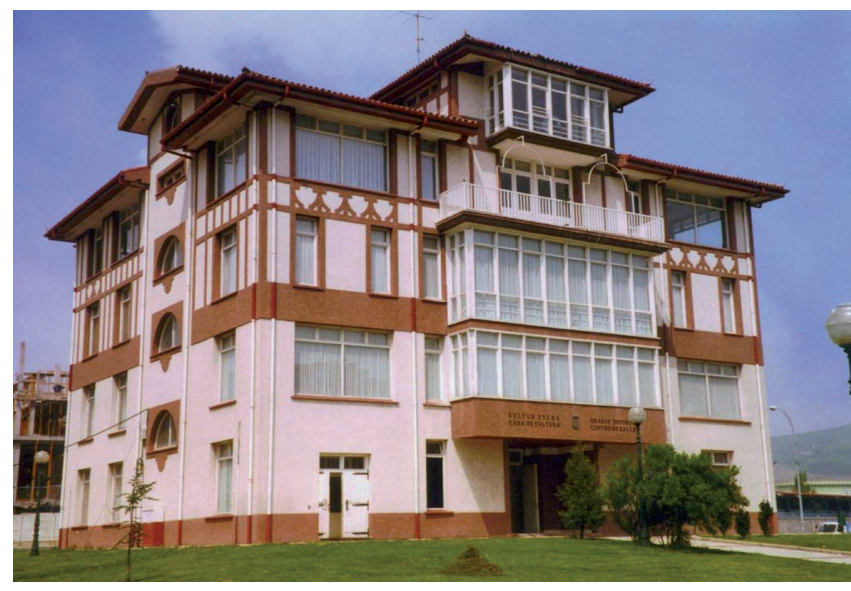

Casa de Cultura de Muskiz. La biblioteca está en la primera planta

Analizar cómo ha evolucionado la adquisición y el uso de la tecnología empleada en este centro desde su creación (1987) hasta la actualidad nos puede ayudar a comprender los diferentes estadios de la relación biblioteca pública/tecnología y su capacidad para innovar.

\section{a. Finales de los años 80 y primeros 90 . Acceso al ordenador y al SIGB.}

Esta época es la de la tecnología deseada pero impuesta de arriba a abajo. El abaratamiento de ordenadores y programas facilita el acceso a los sistemas integrados de gestión bibliotecaria (SIGB). Las bibliotecas de pequeña escala empezamos a acceder con dificultades a esas herramientas gracias al apoyo de centros de decisión jerárquicamente superiores (en Euskadi el Servicio del Libro y Bibliotecas del Sistema Nacional de Bibliotecas de Euskadi, SNBE) que son quienes establecen las condiciones de utilización de la tecnología: deciden la compra del SIGB, los permisos, parámetros, etc. Es una tecnología para facilitar la realización de procesos técnicos relacionados con el soporte papel (catalogación, circulación, etc.) que supone una mejora innegable en nuestras condiciones laborales.

Todo ello repercute positivamente tanto en el servicio que ofrecíamos a nuestros usuarios como en la imagen de la biblioteca. Esta mejora en el propio centro no consigue romper el tradicional modelo de bibliotecasislas: se facilitan las tareas pero éstas no repercutían en otros centros. Las bibliotecas gestionamos soportes impresos y ofertamos los servicios "tradicionales" a usuarios muy locales y fácilmente identificables. Muskiz se automatiza en 1995.

\section{b. Transición XX/XXI. Expansión de internet: tec- nología deseada de libre elección.}

Internet facilita, por primera vez y de una manera efectiva, el acceso de la biblioteca de pequeña escala a una tecnología de su elección, poniéndola en disposición de ejercitar su capacidad de innovar. Se aplican las primeras innovaciones por el uso abajo-abajo.

Podemos destacar tres factores de impacto:

- El correo electrónico y las listas de distribución profesionales que facilitan un intercambio de ideas más rápido y fluido.

- La posibilidad de capturar catalogaciones (Z39.50) que permite la reutilización del trabajo de otros centros en beneficio propio.

- La aparición de las webs bibliotecarias y sus formas de mostrar y generar servicios que abren nuevas expectativas de servicios y productos.

Es evidente que los servicios tradicionales de la biblioteca están cambiando: junto al papel aparecen nuevos soportes; los conocimientos informáticos son necesarios para gestionar el SIGB; en internet salen recursos de gran valor informativo; el correo electrónico y las webs posibilitan la existencia de usuarios no presenciales, etc. El uso de la tecnología nos abre expectativas y plantea retos comunes que nos afectan como colectivo. Surge (ahora sí) una incipiente conciencia de 
red bibliotecaria y una necesidad de comunicación rápida y flexible entre las diferentes bibliotecas. En esta fase las iniciativas verticales conviven con las horizontales y no siempre coinciden sus intereses.

En 2004 los profesionales de las bibliotecas vascas demandan la creación de un foro virtual al Servicio de Bibliotecas; éste no responde (¿ve una amenaza en el foro o su tamaño no le permite reaccionar a tiempo?) y son las propias bibliotecas las que crean Udalbib, la lista de distribución de las bibliotecas públicas de Eus$\mathrm{kadi}^{3}$. Es un hito de innovación por el uso abajo-abajo en Euskadi: las bibliotecas éramos usuarias de listas de distribución y asimilamos una tecnología en beneficio propio.

\section{c. Siglo XXI. Eclosión de la web social: tecnología deseada y bajo control.}

En la fase actual se acelera la discontinuidad tecnológica, y se producen varios fenómenos significativos, como la popularización de nuevas herramientas de creación/difusión de la información en la web, la consolidación de la figura del usuario como elemento clave que enriquece el sistema de información global, la aparición de información relevante disponible en la web pero no en nuestros catálogos, etc. El mundo bibliotecario ya no concibe la biblioteca sin internet y la web social provoca su reflexión (Arroyo, 2007): nuestras destrezas tradicionales, válidas para los recursos impresos, son insuficientes; es necesario dotar a la biblioteca (tanto física como virtual) de un espacio más interactivo y colaborativo.

Hay dos factores a tener en cuenta sobre la web:

- Tecnológico: la proliferación de herramientas de edición web gratuitas y de muy bajo coste de aprendizaje facilitan el acceso a la publicación web. La biblioteca no necesita el permiso de una instancia jerárquica superior para acceder como usuario de pleno derecho a una red inmersa en un período de discontinuidad tecnológica.

- Mental: la diferencia entre un internauta de la web de los primeros tiempos y uno actualmente parece estar no tanto en las herramientas técnicas en sí como en el uso, que no se limita a la aplicación de las habilidades previstas por los que las concibieron (Bosch, 2007).

El profesional tiene cada vez una mayor destreza en el uso de las herramientas web y las utiliza en función de su experiencia, necesidad y creatividad al margen de la utilización prevista. La tecnología web permite la innovación por el uso y facilita los procesos creativos (González, 2007) a diferencia de la "tecnología SIGB" que, centrada en la gestión del papel y anclada en el modelo tecnológico industrial, no facilita el uso de la creatividad en la búsqueda de nuevas soluciones y debe redefinir su respuesta ante la discontinuidad tecnológica.

\section{“El profesional tiene cada vez una mayor destreza en el uso de las herramientas web y las utiliza en función de su experiencia, necesidad y creatividad"}

El perfil del usuario es cada vez más difuso y no suele encontrar en la biblioteca lo que está acostumbrado a encontrar en la web: la inmediatez y la información en todo tipo de soportes. Frente al modelo informacional clásico (usuario solicita/biblioteca suministra) se percibe otro, bidireccional, en el que usuario y biblioteca se encuentran al mismo nivel. De la biblioteca depende entender esa transición como una oportunidad o como una amenaza. Para una amplia revisión de estos cambios puede consultarse el dossier publicado en la revista Educación y biblioteca (Merlo, 2007).

\section{La biblioteca pública ante la web social: ¿oportunidad o amenaza?}

La biblioteca de Muskiz mantiene una web desde $2002^{4}$ con las limitaciones propias de los centros de pequeña escala: de creación propia, desarrollada con herramientas gratuitas, autoformación mediante experimentación (prueba-error) y autosuficiencia ante la falta de recursos. Estos obstáculos han propiciado un entorno creativo e innovador que nos sitúa en un escenario óptimo para entender y aprovechar las nuevas posibilidades de difusión web.

En 2006 iniciamos un proceso de reflexión para mejorar el mantenimiento de nuestra web y detectamos síntomas preocupantes (Juárez, 2006):

- La actualización de los contenidos nos exigía una mayor inversión de tiempo de trabajo, que debíamos detraer de las actividades tradicionales.

- La falta de páginas web con "motivaciones" similares a las nuestras (de las que poder coger ideas) nos obligaba a ser creativos para desarrollar los servicios; y la creatividad exige tiempo.

- La razón de ser de una biblioteca pública son los usuarios y nuestros servicios web no favorecían la comunicación con ellos.

- En nuestra web hay información muy relevante para nuestra comunidad local que no podíamos suministrar a través de nuestro catálogo. Además mucha de esa información estaba siendo generada por la propia biblioteca: artículos, vídeos, fotos.

Con los recursos disponibles no podíamos mantener/generar servicios y las herramientas "tradicionales" (SIGB) marginaban información, sobre todo de ámbito 


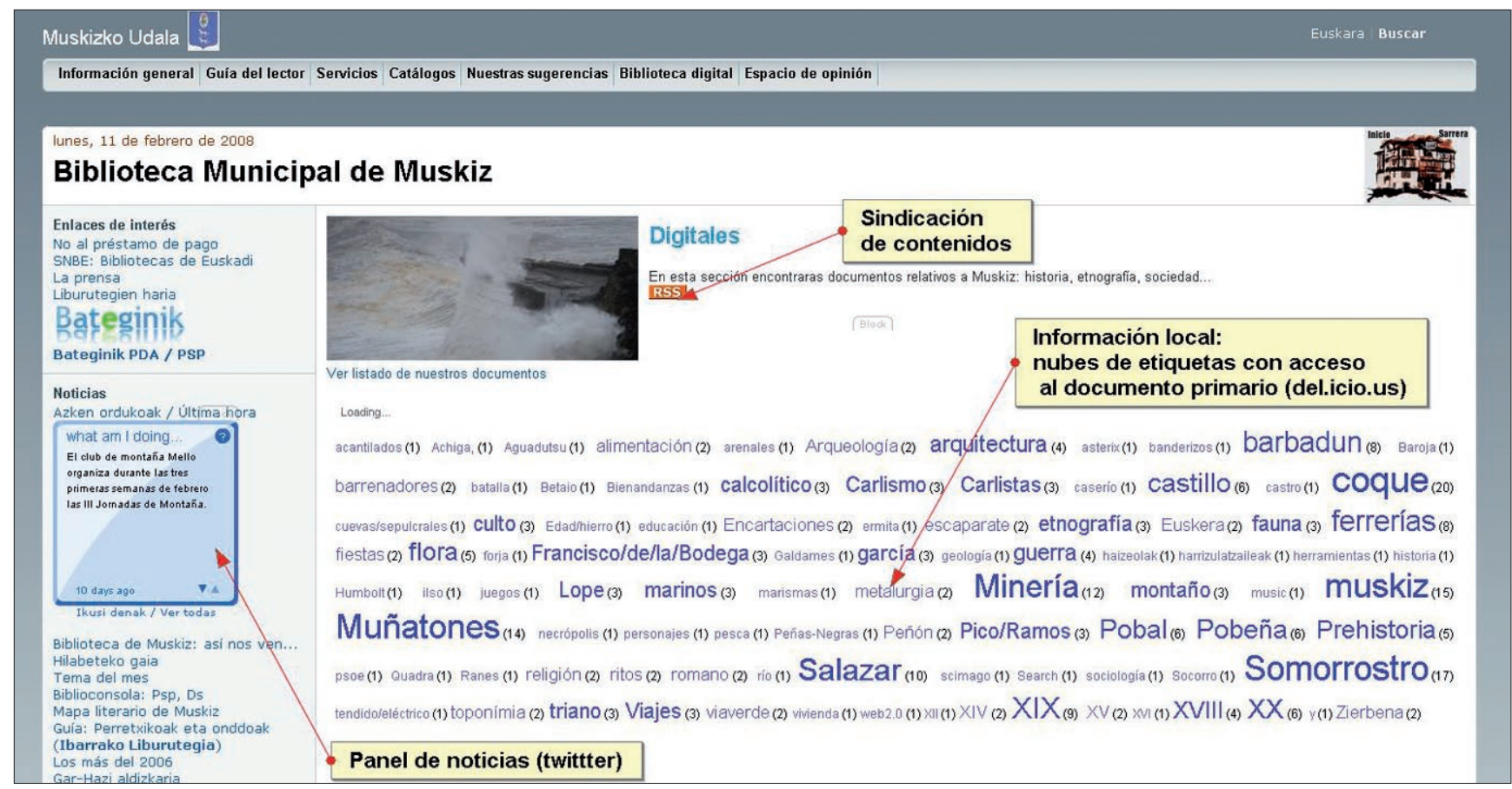

Integración de herramientas web en la biblioteca: etiquetado, microblogging y sindicación

local, imprescindible para una biblioteca pública. El aumento de recursos era implanteable y el esquema tradicional con respuesta arriba-abajo no respondía a nuestras necesidades. Nuestra biblioteca por sí sola no tenía posibilidad de revertir la amenaza tecnológica en oportunidad.

\section{"En 2006 nos dimos cuenta que nuestra biblioteca por sí sola no tenía posibilidad de revertir la amenaza tecnológica en oportunidad"}

Nuestro conocimiento del entorno web nos permite imaginar productos y servicios. Y aunque algunos podemos iniciarlos en solitario (sobre todo los referentes a la información local: nubes de Muskiz, mapa literario, la línea del tiempo, grupo Muskiz, personas y lugares en Flickr, integración del catálogo en el navegador, tablón de anuncios con microblogging, etc.) sabemos que necesitamos ayuda para abordar nuevos servicios con garantías. Invertir dinero, tiempo y mucho trabajo en desarrollar mashups $s^{5}$ que no transcienden el ámbito local no es rentable desde un punto de vista social, es desperdiciar los recursos (Cabezas, 2007).

Por tanto, la biblioteca necesita aplicaciones imaginativas y útiles, portales bibliotecarios atractivos que inviten a la colaboración, que fomenten el surgimiento de usuarios participativos. Hay que buscar la escala adecuada para desarrollar proyectos; en España en el ámbito de la biblioteca pública el contexto autonómico (el sistema bibliotecario autonómico) es ideal para implementar este tipo de tecnologías (web colaborativa). En nuestro caso fueron las bibliotecas que integramos el Sistema Nacional de Bibliotecas de Euskadi.

\subsection{Redimensionando la escala bibliotecaria}

La experiencia adquirida utilizando las nuevas herramientas colaborativas podía ayudarnos en dos frentes: encontrar recursos adicionales en otras bibliotecas y romper el "corsé" del SIGB para ofrecer información a los usuarios. Sabemos que la tecnología nos permite la reutilización de los contenidos y que cuantos más usuarios haya implicados en la generación y difusión de un determinado tipo de información, mayores serán las posibilidades que se podrán obtener utilizando la sindicación web ${ }^{6}$. Pero necesitamos que esos contenidos se adecuen a la naturaleza de la biblioteca. ¿Qué usuarios pueden crear algo para una biblioteca pública?, evidentemente otras bibliotecas públicas. Una solución sencilla para dotar de contenidos pertinentes a nuestra web es agregar los de otras bibliotecas, aunque el problema reside en que hay muy pocas presentes en la web.

\section{1.a. Primer paso. Fomentando la aparición de contenidos afines}

La biblioteca de Muskiz no tiene una posición jerárquica superior sobre las otras bibliotecas de Euskadi, por lo que no puede imponer nada; pero sí puede estimular procesos colaborativos horizontales, abajo-abajo. Es necesario implicar a otros centros en un proyecto común que beneficie a todos los participantes. 
Necesitábamos cambiar ciertos procesos de trabajo en bibliotecas independientes y precisábamos explicar la web social a bibliotecas que no tenían web propia.

Materializar un producto requiere una fase de aprendizaje y prueba de la tecnología que el innovador debe asumir, y nosotros inconscientemente llevábamos tiempo haciéndolo. La lista de distribución Udalbib es un canal de difusión perfecto para llegar a casi todas las bibliotecas de Euskadi. Muskiz es un miembro muy activo: la costumbre de enviar mensajes contando nuestros avances/retrocesos con la tecnología nos dio "prestigio" y cierta capacidad de liderazgo "tecnológico" entre nuestros iguales.

En plena efervescencia 2.0 muchas bibliotecas no disponen de un espacio web propio ${ }^{7}$. Aunando el deseo por tener una página propia con nuestra necesidad de "socios" difundimos un mensaje en el que se animaba a crear un blog y se explicaba cómo utilizar ${ }^{8}$ una de las herramientas más populares $(\text { Blogger })^{9}$. No centramos el mensaje en las virtudes tecnológicas del blog (xml, $R S S$, etc.); en este primer instante nos interesaba difundir un mensaje más simple: hacer una página web es fácil y no hay que pedir permiso. Pretendíamos fomentar la aparición de webs bibliotecarias, futuros "usuarios biblioteca" con los que poder colaborar en la creación y difusión de los contenidos. A modo de ejemplo, y para ver sus posibilidades, se mostraban los blogs de la biblioteca de Muskiz: Pestañas sobre la almohada ${ }^{10} \mathrm{y}$ Txikientzat ${ }^{11}$.

\section{1.b. Segundo paso. Innovación de servicios: $\mathrm{Li}$ - burutegien haria}

Disponer de capacidad de edición en la web es un paso necesario e imprescindible para convertirte en usuario emisor, pero ser "usuario biblioteca" implica utilizar el espíritu colaborativo, ser conscientes de las nuevas reglas de juego y conseguir que el usuario se implique (Juárez, 2007).

Para explicar la sindicación, la agregación y ver las posibles utilidades y beneficios que pueden reportar a las bibliotecas, creamos un agregador cerrado (utilizando ideas y software de otras personas, 11 feeds $s^{12}$ vía Infohilos ${ }^{13}$ ) en el que se reunieron los blogs de las bibliotecas estimuladas por el mensaje difundido en Udalbib. Surgió así Liburutegien haria ${ }^{14}$ (El hilo de las bibliotecas), una web en la que se recogen las novedades de los blogs de manera automática. Esta iniciativa tiene el espíritu de la web colaborativa porque:

- Un usuario (biblioteca de Muskiz) decide agregar y distribuir los contenidos de las páginas que le interesan haciendo uso de los contenidos de otros usuarios.

- Las bibliotecas referenciadas son actores pasivos: han creado unos contenidos susceptibles de ser sindicados y por tanto reutilizados por otros usuarios; su contenido está siendo gestionado por un usuario ajeno y tal vez no sepan que están participando en este proyecto.

- El usuario final recibe un producto muy enriquecido que es sustancialmente diferente al creado por las bibliotecas individualmente.

Con la combinación de la sindicación pasiva de contenidos bibliotecarios en un agregador cerrado obtuvimos un prototipo que nos ha permitido:

- Crear un producto nuevo, de alto valor añadido, que supone una nueva forma de colaboración interbibliotecaria.

- Demostrar que la tecnología web nos permite acometer tareas que cada centro individualmente no podría realizar por falta de medios.

- Adquirir prestigio al llamar la atención de especialistas que han definido el recurso como ejemplo de "biblioteca 2.0" 15 , y de buena práctica en la Administración ${ }^{16}$.

\section{1.c. Tercer paso. Innovación de servicios e innova- ción de procesos: el proyecto Bateginik}

Quien lleve el día a día de una web sabe que lo más difícil es mantener los contenidos actualizados. Nosotros sabemos por experiencia que nuestra escala no nos permite asumir la creación de todos los contenidos que deseamos. Si con Liburutegien haria conseguimos ofrecer contenidos pertinentes a nuestros usuarios, ahora queríamos acometer un proyecto más amplio: propiciar que otras bibliotecas generasen contenidos deseados bajo unas condiciones que permitiesen su redistribución como si fuese un producto de "marca blanca"17.

El reto era ambicioso. Consistía en incidir y modificar los procesos productivos de otras bibliotecas para crear un nuevo producto: innovación de procesos y de servicios. Para ello propusimos un producto práctico, tangible y necesario: la creación de forma cooperativa y descentralizada, de un servicio de novedades bibliográficas accesible desde internet.

La mayoría de las bibliotecas desea ofrecer un boletín actualizado de novedades bibliográficas a sus usuarios pero por diferentes motivos no llega a realizarlo (falta de recursos humanos, de tiempo o de motivación). La tarea, inabordable para una única biblioteca, es ahora posible gracias a la división del trabajo que facilitan las tecnologías. El trabajo previo realizado, fomentando la aparición de blogs bibliotecarios, ha sido fundamental para promover un proyecto en el que la existencia de un número mínimo de bibliotecas en la red con experiencia y sin medios tecnológicos era vital para el impulso inicial. 


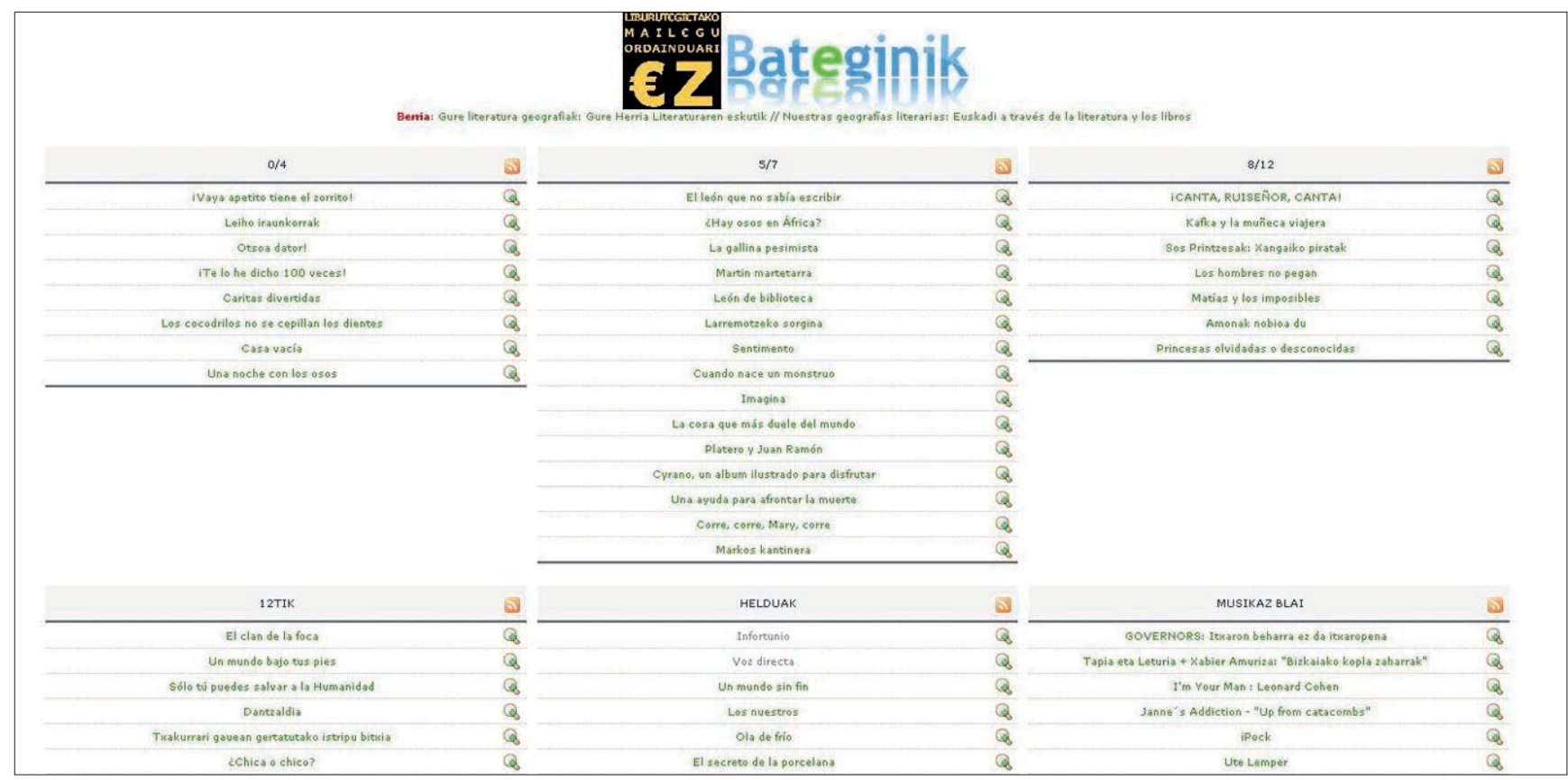

Página de acceso a Bateginik

\section{"Bateginik demuestra que se pueden modificar procesos horizontalmente: la biblioteca pública puede ver y materializar en el actual entorno tecnológico una oportunidad de futuro"}

El estímulo se difundió una vez más por Udalbib. La biblioteca de Muskiz imaginó el producto (agregador, blogs, plantillas) y asumió los costes tecnológicos (creación del entramado de blogs, de la página agregadora, alojamiento en su servidor) y de formación (organización de un taller práctico en el que se explicó cómo funciona un blog y la sindicación de contenidos). Al ser una iniciativa horizontal propuso unas reglas de juego que fueron aceptadas: se planteó un contenido común (reseña de materiales disponibles en nuestras bibliotecas), y un continente que cumpliese cuatro requisitos: título de la obra, imagen, texto alusivo, e incluir el enlace al catálogo del $S N B E$ para obtener información sobre la disponibilidad del recurso. No se establecieron ni criterios de compra previa y/o compartida de materiales, ni plazos de entrega de reseñas, ni restricciones en la creación de contenidos.

Asumido este compromiso mínimo para que el proyecto funcionase, el método de trabajo propuesto, muy al estilo de la filosofía web colaborativa, propugna que cada "usuario biblioteca" sea responsable de la creación de sus contenidos: la decisión de qué material se va a reseñar y cuándo ha de hacerse son decisión exclu- siva de cada centro. Se ha prescindido de aquellas posibilidades técnicas que aún enriqueciendo el producto hubiesen supuesto en los momentos iniciales un posible problema (por eso no se utilizan las categorías ni se etiquetan las entradas). Se decidió en aras de facilitar una mejor difusión no incluir el logo de ninguna de las bibliotecas participantes.

La página de acceso (el mismo agregador cerrado utilizado en Liburutegien haria) recoge automáticamente todas las entradas de los blogs desde el momento en que se producen (sindicación de contenidos facilitada por el propio blog). El usuario final, sea otra biblioteca o un particular, accede a un contenido de calidad, un boletín de novedades actualizado, que le indica a través del catálogo colectivo en qué bibliotecas del sistema tiene disponible la obra.

El producto se denomina Bateginik ("Todos a una" en euskera) y se puso en marcha en octubre de $2006^{18}$. Es la demostración de que se pueden modificar procesos horizontalmente, entre iguales: la biblioteca pública puede ver y materializar en el actual entorno tecnológico una oportunidad de futuro. Más allá del logro tecnológico podemos remarcar el cambio de mentalidad operado en los agentes implicados. A la tradicional estructura arriba-abajo se le añaden iniciativas cooperativas y descentralizadas abajo-abajo que marcan la pauta para futuras colaboraciones. La biblioteca pública, tenga el tamaño que tenga, puede crear y difundir nuevos servicios bibliotecarios.

Bateginik funciona en horizontal pero ha fracasado en vertical (abajo-arriba). Todos los participantes creímos oportuno que la instancia superior, el Servicio de 


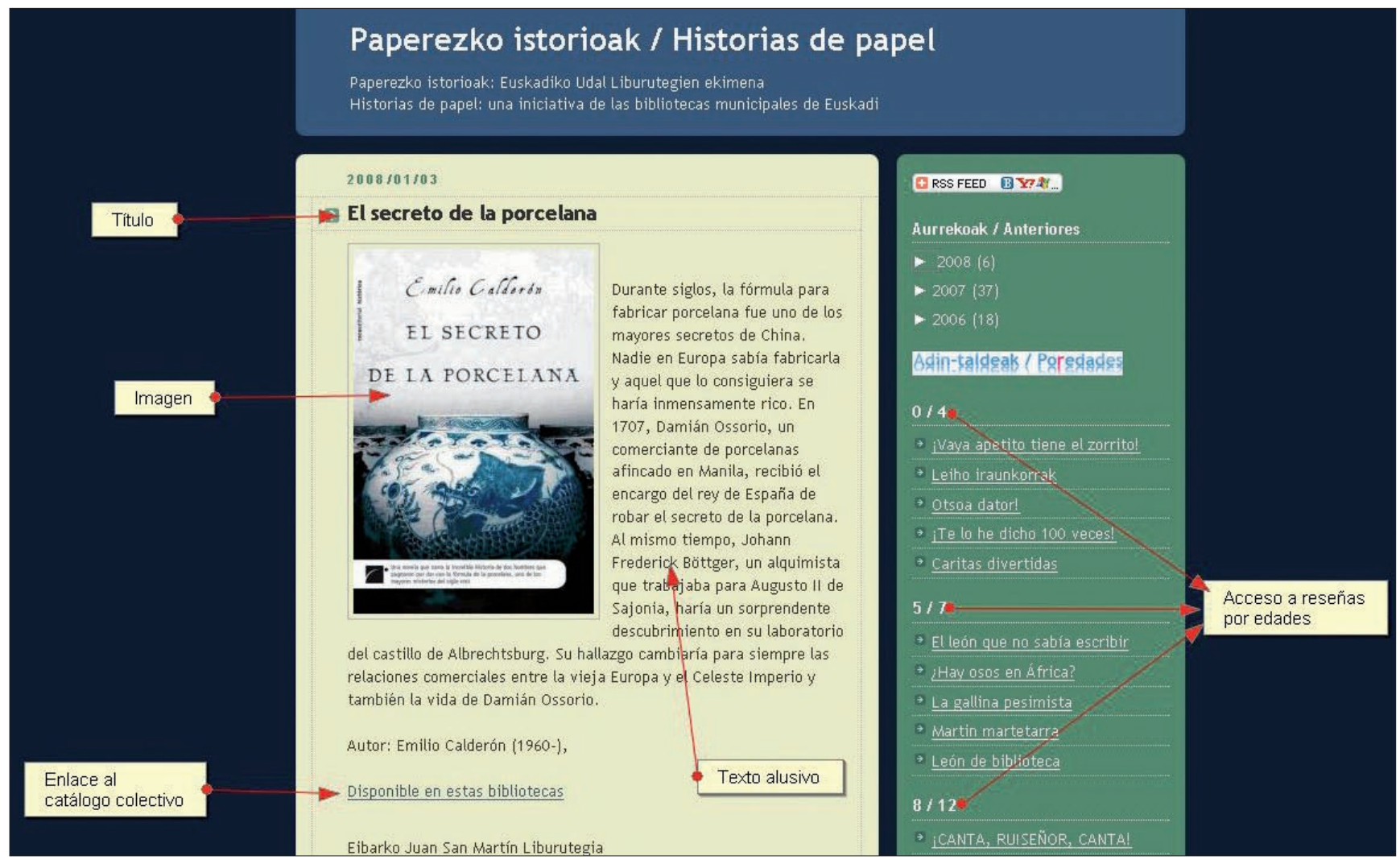

Reseña "tipo" de Bateginik

Bibliotecas del Gobierno Vasco participase en el proyecto. Propusimos a sus responsables que asumiesen el paraguas tecnológico (alojamiento en su servidor, creación y mantenimiento de los blogs con herramientas propias que garantizasen la perdurabilidad y limpieza del producto) y la formación de nuevas bibliotecas colaboradoras. Aún no hemos recibido respuesta. ¿Consideran Bateginik una amenaza o su tamaño no les ha permitido todavía tomar una decisión al respecto?

\section{Conclusiones}

La evolución de las tecnologías de la información, la aparición de nuevos soportes y formas de difundir las ideas hacen necesario un replanteamiento de los servicios tradicionales de la biblioteca, centrados en gestionar información impresa. Gracias a la desaparición de los costes estructurales, los procesos innovadores no dependen tanto de los recursos como de la creatividad y de la facilidad para tomar decisiones en un entorno cambiante. Un mayor número de bibliotecas está en disposición de innovar y crear nuevas estrategias productivas, entre ellas las públicas de menor tamaño, caracterizadas por su escasez de recursos y porque su personal toma y ejecuta las decisiones.

Un replanteamiento de la estructura tradicional arriba-abajo ayudará a asumir que es posible una creación de productos y servicios iniciados y desarrollados horizontalmente. La web de nueva generación (web

\section{"Las bibliotecas públicas de pequeña escala pueden acometer proyectos tecnológicos que renueven los servicios bibliotecarios y beneficien a sus usuarios"}

social colaborativa) nos indica que esa horizontalidad debe aplicarse también a la relación con el usuario: el rol biblioteca/usuario debe acomodarse al nuevo escenario; el usuario no sólo recibe información, también la suministra y por lo tanto deberá ser tratado en consecuencia.

En este sentido varias iniciativas de la biblioteca de Muskiz ${ }^{19}$ muestran cómo bibliotecas públicas de pequeña escala pueden, adoptando un espíritu abierto, innovador y creativo, acometer proyectos tecnológicos que renuevan los servicios bibliotecarios y benefician a sus usuarios. Para ello ha sido fundamental combinar nuestro conocimiento tradicional con el espíritu y las herramientas de la web colaborativa a la hora de gestionar la información de la biblioteca.

\section{Notas}

1.http://www.euskadinnova.net/opencms/export/sites/default/web_euskadi_ innova/es/euskadi_innova/Documentacion/Manual_de_Oslo_es.pdf

2. Mapa de lectura de Euskadi 2007. 
http://www.kultura.ejgv.euskadi.net/r46-4879/es/contenidos/informacion/ mapa_lectura/es_mapa/adjuntos/Mapa_lectura_EAE.pdf)

3. http://es.groups.yahoo.com/group/udalbib/

4. http://www.muskiz-liburutegia.org

5. http://es.wikipedia.org/wiki/Mashup

6. http://es.wikipedia.org/wiki/Sindicaci\%C3\%B3n_web

7. Una de las quejas recurrentes entre los profesionales de las bibliotecas públicas vascas es la dificultad para disponer de una web, bien por dificultades administrativas o tecnológicas.

8. http://www.muskiz-liburutegia.org/biblioteca/blog.pdf

9. http://www.blogger.com/start?hl=es

10. http://betileak.blogspot.com/

11. http://txikientzat.blogspot.com/

12. http://e.leven.com.ar/11feeds/

13. http://www.infohilos.com/

14. http://www.muskiz.com/biblioteca/Sindikazioa/index.php

15. Biblioblog. Bibliotecas 2.0. Consultado en: 09-12-07.

http://www.interreg-eet.info/weblogs/biblioblog/archives/001095.html

16. Administraciones en red. Sindicación de contenidos en bibliotecas vascas. Consultado en: 09-12-07.

http://eadmin.blogspot.com/2006/05/sindicacin-de-contenidos-de.html

17. También llamada marca de distribuidor, es la marca perteneciente a una cadena de distribución (generalmente, hiper o supermercado) con la que se venden productos de distintos fabricantes. El fin de la marcas blancas es conseguir una fidelización para los productos del comercio. En: Wikipedia. Marca blanca. Consultado en: 09-12-07. http://es.wikipedia.org/wiki/Marca_blanca

18. http://www.muskiz-liburutegia.org/biblioteca/Sindikazioa/bateginik.php 19. http://www.muskiz-liburutegia.org/informes.html

\section{Bibliografía}

Arroyo, Natalia. “¿Web 2.0? ¿web social? ¿qué es eso?”. En: Educación y biblioteca, 2007, v. 19, n. 161, pp. 69-74. Consultado en: 06-02-08. http://eprints.rclis.org/archive/00011752/01/EYB_NA07.pdf

Bosch, Mela. "La indización asistida para el manejo de conocimiento". En: Entre la heurística y la hermenéutica: las competencias y actitud de los profesionales de la información para la web emergente. Documentalistas. org. Consultado en: 09-12-07.

http://eprints.rclis.org/archive/00009888/01/BoschMelaHeurYHermenWeb Emergente.pdf
Cabezas, Álvaro. "La biblioteca 2.0 y las escalas" En: Documentación, biblioteconomía e información, 8 de marzo de 2007. Consultado en: 0912-07.

http://www.lacoctelera.com/documentacion/post/2007/03/08/la-biblioteca2-0-y-escalas

EAEko irakurketa publikoaren mapa = Mapa de lectura pública en la $C A P V$. Vitoria-Gasteiz: Eusko Jaurlaritzaren Argitalpen Zerbitzu Nagusia = Servicio Central de Publicaciones del Gobierno Vasco, 2007.

Freire, Juan. "Sobre la sociedad de los intangibles: creatividad, ciudadanos y políticos". En: Nómada, 10 de octubre de 2007. Consultado en: 09-1207.

http://nomada.blogs.com/jfreire/2007/10/sobre-la-socied.html

González-Fernández-Villavicencio, Nieves. "Bibliotecas de nueva generación: bibliotecas 2.0”. En: Educación y biblioteca, 2007, v. 19, n. 161, pp. 75-84. Consultado en: 06-02-08.

http://eprints.rclis.org/archive/00012029/01/Biblioteca20.PDF

Jantz, Ronald C. "Innovaciones tecnológicas en la biblioteca: proyectos digitales que ofrecen nuevas oportunidades al bibliotecario y a la biblioteca". En: Boletín de la Asociación Andaluza de Bibliotecarios, 2002, v. 67, pp. 61-69. Consultado en: 09-12-07.

http://eprints.rclis.org/archive/00003185/01/67a4.pdf

Juárez-Urquijo, Fernando. "La biblioteca pública, un usuario más de la web 2.0". En: III Congreso nacional de bibliotecas públicas, 2006. Consultado en: 09-12-07.

http://www.muskiz-liburutegia.org/Doc/web\%2020\%20y\%20bibliotecas. $p d f$

Juárez-Urquijo, Fernando. "La web 2.0 en una biblioteca pública". En: Educación y biblioteca, 2007, v. 19, n. 161, pp. 103-112. Consultado en: 06-02-08.

http://www.muskiz-liburutegia.org/dok/ebweb20.pdf

Merlo-Vega, José-Antonio (coord.). "Dossier bibliotecas y web social". En: Educación y biblioteca, 2007, septiembre-octubre, v. 19, n. 161, pp. 62-124.

Sánchez-Novoa, Enrique. "Visión estratégica de la innovación empresarial". En: Revista Madri+d, 2002, agosto-septiembre, v. 12. Consultado en: 09-12-07.

http://www.madrimasd.org/revista/revista12/aula/aulas1.asp

Fernando Juárez-Urquijo, Biblioteca municipal de Muskiz, Kultur Etxea, Cendeja 29, Muskiz, 48550 Bizkaia.

ferjur@gmail.com

liburutegia@muskiz.com

http://www.muskiz-liburutegia.org/

\section{Próximos temas centrales}

\author{
Mayo 2008 \\ Julio 2008 \\ Septiembre 2008 \\ Noviembre 2008
}

\author{
Presente y futuro de la profesión \\ Libros electrónicos \\ Información en la empresa \\ Redes sociales
}

Los interesados pueden remitir notas, artículos, propuestas, publicidad, comentarios, etc., sobre estos temas a:

epi@elprofesionaldelainformacion.com 